\title{
The action effect: Support for the biased competition hypothesis
}

\author{
Greg Huffman ${ }^{1}$ • Jay Pratt ${ }^{1}$
}

Published online: 7 June 2017

(C) The Psychonomic Society, Inc. 2017

\begin{abstract}
The action effect refers to the finding that faster response times are found when a previously responded to stimulus contains a target item than when it serves as a distracting item in a visual search. The action effect has proven robust to a number of perceptual and attentional manipulations, but the mechanisms underlying it remain unclear. In the current study, we present two experiments investigating a possible underlying mechanism of the action effect; that responding to a stimulus increases its attentional weight causing the system to prioritize it in the visual search. In Experiment 1, we presented the search stimulus in isolation and found no evidence of an action effect. Thus, when there was no requirement for prioritization, there was no action effect. In Experiment 2, we tested whether stimulus-based priming (rather than the action) can account for the observed validity effects. We found no evidence of a priming effect when there were never any actions. These findings are consistent with the biased competition hypothesis and provide a framework for explaining the action effect while also ruling out other potential explanations such as event file updating.
\end{abstract}

Keywords Attention: Selective $\cdot$ Perception and action . Attention and memory

In contrast to the long history of vision and attention research focusing on manipulating stimulus properties and observing the caused behavioral changes, recently there has been a surge of research which manipulates both stimulus and response

Greg Huffman

greg.huffman@mail.utoronto.ca

1 Department of Psychology, University of Toronto, Toronto, Ontario, Canada features. This research has indicated that visual processing and attention bidirectionally interact with response processes across a range of processing levels, a sharp contrast with assumptions of strict modularity across the different systems (Fodor, 1983). Take attention in a visual search task; if a specific color is associated with a specific response, that color is more effectively ignored if it is a distractor and more quickly found if it is a target after that keypress is made (Gozli, Aslam, \& Pratt, 2016; Gozli, Moskowitz, \& Pratt, 2014). In terms of perceptual processing, action can affect perception in several ways, including the visual system being less sensitive to the perceptual consequences of self-produced actions (Blakemore, Wolpert, \& Frith, 1998; Cardoso-Leite, Mamassian, Schütz-Bosbach, \& Waszak, 2010; Müsseler \& Hommel, 1997), and being sensitive to current action affordances in the surrounding environment (Taylor, Witt, \& Sugovic, 2011; Witt, Linkenauger, \& Proffitt, 2012; but see: Firestone \& Scholl, 2015). Indeed, merely presenting stimuli on participants' hands, rather than using a monitor, alters how attention is allocated (Taylor \& Witt, 2014), and placing participants' hands near the monitor, rather than on a keyboard, affects a wide range of visual processes (Abrams \& Weidler, 2013; Gozli, West, \& Pratt, 2012; Huffman, Gozli, Welsh, \& Pratt, 2015; for a review, see: Taylor, Gozli, Chan, Huffman, $\&$ Pratt, 2015). Fitting in with these lines of research is the recently observed "action effect" (Buttaccio \& Hahn, 2011; Wang, Sun, Sun, Weidler, \& Abrams, 2017; Weidler \& Abrams, 2014; 2017), which is the focus of the present study.

In studies reporting the action effect, participants are presented with a color word (written in white) followed by a centrally presented colored shape, such as a blue circle, and are instructed to press the spacebar if the color referred to by the word matches the shape's color. A visual search follows in which participants search for and respond to a tilted line among vertical lines. Importantly, the lines are in colored shape placeholders and one 
of the placeholders always matches the shape and/or color of the item from the first part of the task. If the first stimulus was responded to (action condition) and it then serves as the placeholder for the target line (valid condition), responses are facilitated relative to when the target appears within a non-responded to placeholder (invalid condition). If the second stimulus is not responded to (no action condition), however, no differences between responses times (RTs) in the valid and invalid conditions are typically found.

Buttaccio and Hahn (2011) originally reported the effect in their first experiment, and conducted four additional experiments. In their Experiment 2, they analyzed whether the RT distribution shifted, became more disperse, or changed in skew as a function of action and validity conditions, and found that the action effect was caused by shifts in the distributions rather than variations within the distribution. In their Experiment 3, they tested whether the repetition of a given color across the stimulus displays was causing the participants to adopt an attentional set for that color, leading to the validity effect (rather than the action leading to the validity effect). To do so, they had participants make a go response if the go/no-go stimulus's shape (the stimulus also had a color) matched a preceding shape word. Critically, the shape of the go/no-go stimulus was always absent from the search display, but the color was present. If the action effect was due to the participants adopting an attentional set for a repeated stimulus attribute that was relevant to the task, then it should not have been found in that experiment. In contrast to this prediction, the action effect remained. The attentional set hypothesis was further tested in Experiment 4 in which they had participants withhold a response if the color word and the go/no-go stimulus color was the same, but execute the response if they were different. They also included two intervals between the go/no-go stimulus and the search display (100 and $500 \mathrm{~ms}$ ). At the short interval, they found an effect of stimulus repetition with faster responses to valid relative to invalid targets in the no action condition where the stimulus color repeated across stimulus displays. They also found that this effect dissipated quickly by finding the more typical (though reduced) action effect at the $500 \mathrm{~ms}$ interval. In their final experiment, they presented a digit within the go/nogo stimulus and had participants respond if it matched a preceding digit (such that they were making an action to a stimulus feature completely absent from the search display). Despite this change, they once again found the action effect with faster responses to valid compared to invalid targets in the action condition. The authors interpreted these data as evidence that responding to a stimulus strengthens that stimulus's memory trace which leads to its attentional weight being increased. This increased attentional weight causing that stimulus to win the competition for selection during the search phase which manifests as a RT validity effect.

Weidler and Abrams (2014) reported four experiments in which they manipulated how the go/no-go response was determined and the timing of the go/no-go stimulus offset. In their first experiment, they replicated the Buttaccio and Hahn (2011) study using two rather than six search items. In Experiment 2, they changed the timing of the go condition such that it was the same as the no-go condition (whereas previously it offset once a response was made). In Experiment 3, they gave simple go/no-go instructions rather than using the matching procedure. Finally, in Experiment 4, they removed the action cue word and presented an ' $\mathrm{X}$ ' within the go/no-go stimulus on half of the trials. They instructed the participants to make a spacebar response if there was no ' $\mathrm{X}$ ' in the stimulus and to withhold the response if the ' $\mathrm{X}$ ' was present. In all four experiments the authors observed the action effect. Moreover, in Experiment 4, they observed both an action effect and a reversed action effect with faster responses to invalid compared to valid targets in the no-go conditions. The authors propose multiple candidate hypotheses regarding the action effect including event files (Hommel, 1998; Hommel, Müsseler, Aschersleben, \& Prinz, 2001) in working memory guiding search, feature repetition priming (Maljkovic \& Nakayama, 1994), and the biased competition between stimuli (Desimone \& Duncan, 1995). Taken together with the Buttaccio and Hahn (2011) findings, the action effect appears markedly robust to an array of perceptual and attentional manipulations.

Before detailing the current study, we need to point out two subtle but important differences between the studies of Buttaccio and Hahn (2011) and Weidler and Abrams (2014). Specifically, the former used four search items (one target and three distractors) and consistently found larger action effects than the latter who used two search items (one target and one distractor) search. Moreover, the large magnitude of the action effect in Buttaccio and Hahn (2011) appears to be due to slower responses in the action - invalid condition rather than faster responses in the action - valid condition (which are approximately equal across the two set sizes). Thus, it appears that making an action to a stimulus prioritizes that stimulus for attentional selection in the search display so that it will be searched first (see: Wang et al., 2017, for evidence for this from eye movements). This would make the set size irrelevant in the action - valid condition, as the valid item will be searched for first regardless of search set size. In contrast, in the invalid condition, the distractor matching the go/no-go stimulus will be searched first and then the remaining items will be searched inefficiently. This will lead to larger validity effects with larger set sizes because there are multiple remaining items to search through. When there is a set size of two, the target item can immediately be found after searching a distractor, thus yielding smaller validity effects.

Taken together, the differences between the two previous studies (Buttaccio \& Hahn, 2011; Weidler \& Abrams, 2014) lead us to believe that the most likely of the previously proposed hypotheses for the action effect is the biased 
competition principle. According to this principle, behaviorally relevant stimulus features are weighted more heavily by the attentional system than behaviorally irrelevant features causing them to win the competition for attentional selection (Bichot, Rossi, \& Desimone, 2005; Desimone \& Duncan, 1995). While both of the previous studies speculated that the biased competition principle may underlie the action effect, we directly test this hypothesis in the current study. Specifically, there is a clear prediction if the biased competition hypothesis of the action effect is correct; removing the search component of the task should abolish the action effect. This is because, as the name suggests, biased competition accounts for which of the available stimuli will be selected for further processing. In the absence of competing stimuli, no biases will be applied to any presented stimulus.

In the current study, we pit the biased competition prediction against that from an alternative hypothesis; the action effect is due to the processes of feature integration that occur with event file representations. Specifically, it is possible that an abstract stimulus-response binding is formed for the prime stimulus. This stimulus-response binding would need to be abstract (e.g., a "respond" code integrated with a stimulus) because the response sets between the prime and search tasks do not overlap and the action effect has been shown to be cross-modal (Wang et al., 2017). Once a prime stimulus has been bound with the response code, there will be facilitation if a response is required to that stimulus during the search task. Alternatively, if the "respond" code is assigned to a stimulus other than the one the search target appears in, there may be a cost to responding (Hommel, 1998).

In terms of the current study, the event file hypothesis differs from the biased competition hypothesis in an important way. In the absence of distracting stimuli, the biased competition hypothesis predicts there will be no action effect. The lack of distracting competition, however, is not a problem for the event file account. Feature integration effects are regularly found when using isolated stimuli (Hommel, 1998; Hilchey, Rajsic, Huffman, \& Pratt, 2017; Huffman, Al-Aidroos, \& Pratt, 2017) even when attention is occupied in an alternative task (Hommel, 2005). Thus, if feature integration underlies the action effect, the action effect should still be present when no distracting stimuli are presented for the search task.

We test the biased competition and feature integration hypotheses against each other in Experiment 1. To do so, we have one group of participants complete a typical action effect task and a second group of participants complete the same task except there was no distractor stimulus in the second part of the task. According to the biased competition hypothesis, the action effect should be absent in this no search group. In Experiment 2, we further test whether the action effect may be due to stimulus repetition priming by showing participants the same stimuli as typical action effect experiments, but not having participants make responses to them (see: Buttaccio \&
Hahn, 2011, Experiments 3 and 4). If feature integration underlies the action effect we would expect to find a cost for having to respond to a stimulus that a response was withheld from previously. This is because previous research has shown that a "no response" code can be integrated with stimulus codes (Kühn \& Brass, 2010; Kühn, Elsner, Prinz, \& Brass, 2009; Röttger \& Haider, 2016) such that responding to that stimulus will generate a cost. The biased competition hypothesis predicts no benefit from merely viewing the prime stimulus.

\section{Experiment 1}

We looked to compare the biased competition hypothesis of the action effect to the feature integration hypothesis. To that end, we had one group of participants complete a constructive replication (Haig, 2005) of previous action effect studies and compared their performance with another group of participants that saw no distractors during the search phase (i.e., only the target stimulus and its placeholder were presented). Our procedure most closely matched Weidler and Abrams's (2014) Experiment 1, with the difference being that we used two rather than six colors. To recap, the biased competition hypothesis predicts that removing the distractors from the search will eliminate the action effect while the feature integration account predicts the action effect will be present in the no search condition. Thus, finding an interaction between the two groups where there is no action effect in the no-search condition would be support for the biased competition hypothesis. Finding the action effect in both conditions would support the feature integration hypothesis.

\section{Method}

Participants Thirty-four undergraduates (17 in each condition) from the University of Toronto participated in the experiment and were compensated with course credit. All participants provided informed consent, were naïve to the experiment's purpose, and reported normal or corrected-to-normal visual acuity and color vision.

Stimuli and apparatus The stimuli and apparatus were the same for the replication and no search conditions. The experiments were conducted on a PC connected to a CRT monitor (screen resolution: $1024 \times 768$; refresh rate: $85 \mathrm{~Hz}$ ). Stimuli were created and presented using Matlab by Mathworks with the Psychophysics toolbox (Kleiner et al., 2007). Stimuli consisted of blue [CIE: luminance $=12.2 \mathrm{~cd} / \mathrm{m}^{2}, \mathrm{x}=0.398$, $\mathrm{y}=0.459$ ] and yellow [CIE: luminance $=10.2 \mathrm{~cd} / \mathrm{m}^{2}, \mathrm{x}=$ $0.361, y=0.459]$ circles subtending $2^{\circ}$ visual angle and black lines subtending $1.6^{\circ}$ visual angle and were presented on a black background. Responses were made using the spacebar 


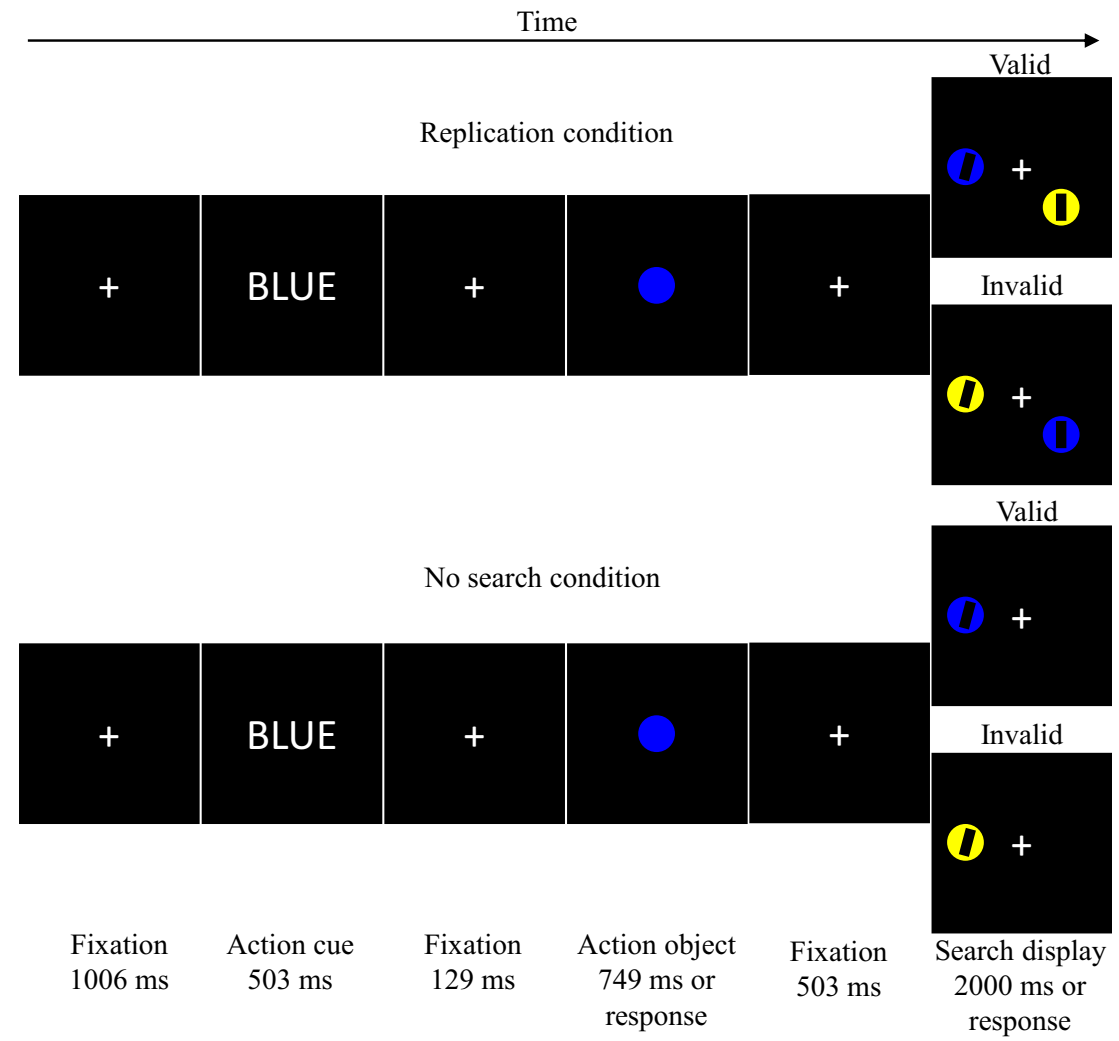

Fig. 1 Action-effect procedure stimuli for the replication and no search conditions. Participants are presented with a color word followed by a colored circle. If the circle's color matches the color word, they make a

and left and right arrow keys on a standard QWERTY keyboard.

\section{Procedure}

Replication condition See Fig. 1 for the experimental stimuli and procedure. Each trial began with a fixation cross which remained for 1,006 ms. Following the fixation cross, we presented a color word ("YELLOW" or "BLUE") in white text. After $503 \mathrm{~ms}$, we re-presented the fixation cross for $129 \mathrm{~ms}$, followed by a blue or yellow circle in the center of the display. We instructed participants to press the spacebar with their left hand if the colored circle matched the color word that preceded it (e.g., the word BLUE followed by a blue circle), but to withhold their response if the color word and circle stimulus did not match (e.g., the word BLUE followed by a yellow circle). After a correct response in the go condition or $749 \mathrm{~ms}$ in the no-go condition, the go/no-go stimulus offset ${ }^{1}$ and a fixation stimulus appeared. After $503 \mathrm{~ms}$, two circles

\footnotetext{
${ }^{1}$ This does create a consistent timing difference between the action and no action conditions since the stimulus offsets upon a response in the action condition. Weidler and Abrams (2014), however, have demonstrated that the action effect remains if this timing difference is eliminated. Given that, we have chosen to maintain the method used by the other action effect experiments.
}

spacebar response. Next, they search for a tilted line and report whether it is oriented to the left or right using the arrow keys. In the no search condition, the display contained only one item.

(one yellow and one blue) were presented in two of five randomly selected locations around an imagery $12^{\circ}$ radius circle (from the fixation cross). Within each circle, we presented a line, with one line being vertical while the other was oriented $5^{\circ}$ to the left or right. We instructed participants to find the tilted line and report its orientation using the left and right arrow keys with their right hand as quickly as they could, without sacrificing accuracy. If at any point during a trial participants made an error we presented "MISTAKE" in red text for $2000 \mathrm{~ms}$ and the trial terminated and was counted as an error.

No search condition The procedure for the no search condition matched that of the replication with one modification: there was no distractor placeholder or line presented during the search phase.

Design Participants first completed a set of 10 practice trials which were repeated if the experimenter deemed it necessary. This was followed by 160 randomly ordered test trials generated by repeating the color word ("YELLOW" or "BLUE"), go/no-go stimulus color (yellow or blue), target placeholder color (blue or yellow), and target line tilt (left or right) 10 times each. For analyses, trials were coded by whether they were an action or no action trial, whether the first circle was a 
valid or invalid cue in terms of which color placeholder the target would appear in, and whether it was the replication or no search condition resulting in a 2 (condition: replication or no search) $\times 2$ (action or no action) $\times 2$ (valid or invalid $)^{2}$ design

\section{Results}

Any trials where participants made an error or responded in less than $100 \mathrm{~ms}$ (for either the first or second response) were trimmed for analyses totaling $11.4 \%$ of the trials. We then conducted a repeated measures, mixed $2 \times 2 \times 2$ ANOVA with action (action or no action) and validity (valid or invalid) as within subjects factors and condition (replication or no search) as a between subjects factor and RTs and error rates as dependent measures (Fig. 2). With RTs as the dependent measure, we observed no main effect of action, $F(1,32)=2.107, M S E=$ $946.925, p=0.156, \eta_{p}^{2}=0.062$, or validity, $F(1,32)<1, M S E=$ 208.717, $p=0.470, \eta_{p}^{2}=0.016$. Condition interacted with action, $F(1,32)=6.041, M S E=2715.793, p=0.020, \eta_{p}^{2}=$ 0.156 , with faster responses following actions in the replication condition, but not in the no search condition. Condition did not interact with validity, $F(1,32)=2.041, M S E=$ 798.214, $p=0.163, \eta_{p}^{2}=0.060$. Critically, however, the three-way condition $\times$ action $\times$ validity interaction was significant, qualifying the above effects, $F(1,32)=11.399, M S E=$ $7129.083, p=0.002, \eta_{p}^{2}=0.263$. To follow-up this interaction, we conducted 2 (action) $\times 2$ (validity) ANOVAs within each search condition. We found no action $\times$ validity interaction in the no search condition, $F(1,16)<1, M S E=134.732, p=$ $0.890, \eta_{p}^{2}=0.001$, but did find the interaction in the replication condition, $F(1,16)=12.429, M S E=13871.592, p=.003, \eta_{p}^{2}=$ 0.437 . Paired $t$ tests (two-tailed, $\alpha=0.05$ ) comparing the validity effect in the action and no action condition found significantly faster response times in the valid $(M \pm \mathrm{SE}$ : $681.79 \pm 17.59 \mathrm{~ms})$ compared to the invalid condition $(703.03 \pm 18.33 \mathrm{~ms}), t(16)=2.556, p=.021, d=0.62,95 \%$ CI $[3.63,38.86]$, in the action condition, replicating the action effect. In the no action condition, surprisingly, we observed an inverse validity effect with faster responses in the invalid condition $(688.68 \pm 22.18 \mathrm{~ms})$ compared to the valid condition $(710.03 \pm 21.30 \mathrm{~ms}), t(16)=2.840, p=0.012, d=0.69,95 \%$ CI [5.55, 38.25].

To follow up these findings, we conducted a Bayesian repeated measures ANOVA using JASP statistics software (JASP Team, 2017) with the same factors as above and using a weakly informative prior, allowing us to compare the

\footnotetext{
${ }^{2}$ It should be noted that, while we have conditions labeled as invalid in both the replication and no search condition, in the replication's invalid condition the go/no-go stimulus appeared within the search display, but in the no search's invalid condition this was not the case.
}
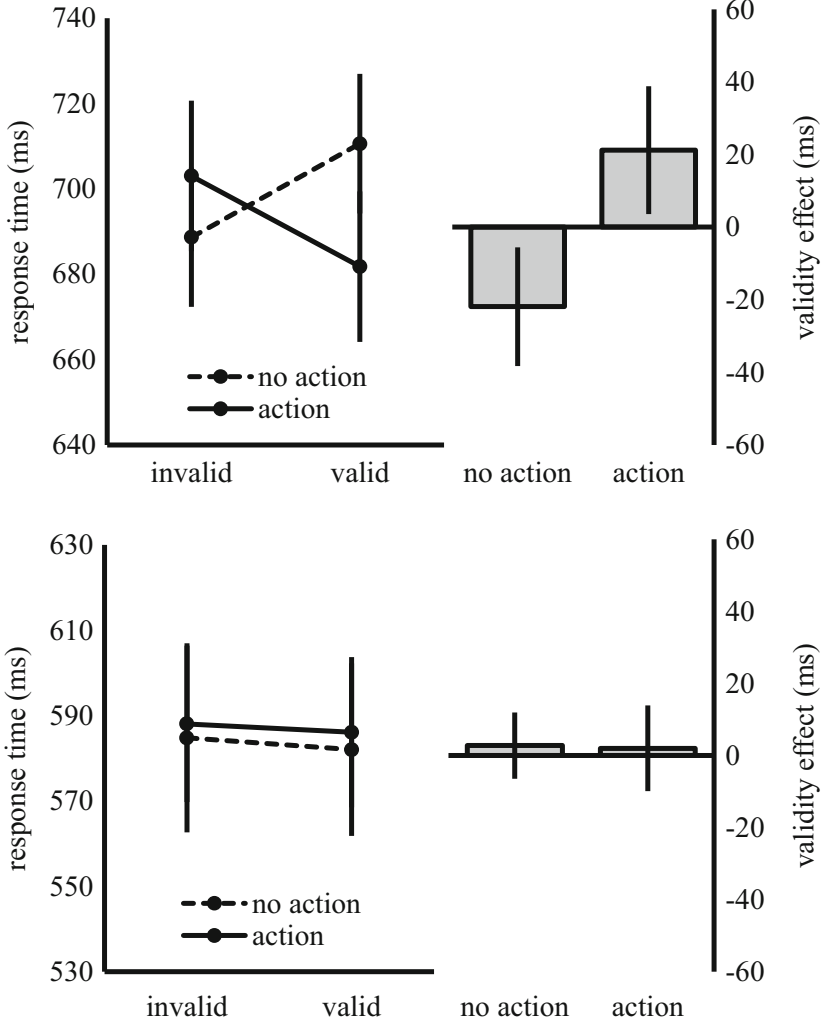

Fig. 2 Results from the replication (top panel) and no search (bottom panel) conditions from Experiment 1. Left panel: RTs as a function of action and validity condition. Right panel: validity effect (invalid RT valid RT) as function of action condition. Error bars represent the $95 \%$ confidence interval of validity effect in both plots.

likelihood of a given model (including the null model with no factors included) relative to another model. We report Bayes factors $(B F)$ which refer to how many times likelier a model is compared to another (e.g., $B F=12$ means that a model is 12 times more likely than a competing model). Most relevant to our current concerns, the analysis most favored the model including the three-way, condition $\times$ action $\times$ validity interaction $(B F>1000)$. In addition, the analysis preferred the three-way interaction model over the model including all terms except for the three-way interaction (all main effects and two-way interactions; $B F=98.98$ ). Similarly, the full three-way model was clearly preferred over the second most highly preferred model (which includes the action and validity main effects, the two-way interaction between them, and a main effect of condition; $B F=47.56$ ). Overall, these results provide strong to very strong evidence for the presence of a three-way interaction (Raftery, 1995).

Following up these analyses, we ran two-way Bayesian repeated measures within the replication and no search conditions. In the replication condition, the analysis strongly preferred the two-way interaction model $(B F=168.65)$ compared to the null model and to the model containing only the main effects $(B F=430.23)$. Next, we conducted Bayesian $t$-tests comparing the null hypothesis of there being no difference 
between validity conditions with the alternative hypothesis of the RTs being different between validity conditions. The data are more likely under the alternative hypothesis in the no action condition $(B F=10.05$ and in the action condition $(B F$ $=2.92$ ). Both of these values represent positive ${ }^{3}$ evidence in favor of there being differences between the invalid and valid conditions.

In contrast, within the no action condition, the analysis failed to find evidence supporting the action effect and instead strongly supported the null hypothesis ( $B F$ $=31.25)$. The null hypothesis was also supported over an action main effect $(B F=2.99)$, a validity main effect $(B F=3.62)$, and independent contributions from both main effects $(B F=31.25)$. Taken together the analysis strongly supported the null hypothesis compared to the action effect and provided positive to strong support for the null hypothesis relative to the main effects.

In the error rate data, we found an interaction between validity and condition, $F(1,16)=6.467, M S E=0.001, p=$ $0.016, \eta_{p}^{2}=0.168$. Slightly lower error rates in the replication condition for responding to valid $(4.46 \pm 0.73 \%)$ compared with invalid (5.85 $\pm 1.14 \%), t(16)=1.428, p=0.173, d=0.35$, $95 \%$ CI $[-0.67,3.44]$, targets and significantly lower error rates in the no search condition for responding to invalid $(5.29 \pm 1.03 \%)$ compared with valid $(7.21 \pm 1.15 \%), t(16)=$ $2.565, p=0.021, d=0.62,95 \%$ CI $[0.33,3.51]$, targets drove this interaction. We found no other main effects or interactions, $F_{\mathrm{S}}<2.067 p \mathrm{~s}>0.160$. These effects were in the same direction as the RT effects and do not represent a speedaccuracy tradeoff. Furthermore, the corresponding Bayesian repeated measures favored the null hypothesis $(B F=11.19)$, further indicating that the RT effects were not related to corresponding accuracy fluctuations. Because the Bayesian analysis strongly preferred the null hypothesis model, we will refrain from interpreting the accuracy effects.

\section{Discussion}

Consistent with the biased competition hypothesis (Buttaccio \& Hahn, 2011; Weidler \& Abrams, 2014), but not the feature integration hypothesis (Wang et al., 2017), the results from the replication condition sharply contrasted with the no search condition. In the replication condition, both the frequentist and Bayesian analyses supported the notion that there was a validity effect in the action condition, consistent with previous action effect experiments (Buttaccio \& Hahn, 2011; Weidler

\footnotetext{
3 The Bayes factor in the action condition does not quite reach the positive evidence value (3), we believe this to be a legitimate effect (though possibly on the smaller end) given the robustness of action effects reported by previous studies as well as our own experience using this paradigm in additional (yet to be reported) experiments.
}

\& Abrams, 2014). In addition, we observed an inverse validity effect in the no action condition, which was also supported by both analyses. This finding is somewhat uncommon, but not completely novel (e.g., Experiment 4, Weidler \& Abrams, 2014). We speculate that an important difference could be that two colors were used rather than six, making it possible that the alternative color is weighted in the opposite direction as the go/no-go color resulting in the observed pattern of results. Indeed, the polarity correspondence principle (Proctor \& Cho, 2006) predicts this would be the case when binary stimuli are used such that each stimulus can be rapidly categorized as the positive or negative stimulus and the biased competition account of repetition priming suggests that distractors are downweighted (Ásgeirsson, Kristjánsson, \& Bundesen, 2015).

Importantly, if attentional weighting through biased competition accounts for the action effect, we predicted that removing the search display would fail to produce an action effect, because removing the search would also remove competition for attentional selection. Supporting this hypothesis, the frequentist analysis failed to reject the null hypothesis and, critically, the Bayesian analysis strongly favored the null hypothesis over the two-way interaction predicted by the action effect. That is, when we presented no distractors during the search phase there was no semblance of a validity or inverse validity effect. This suggests that competition for selection is a driving factor in the action effect, which would indicate that responding to stimuli increases their attentional weight.

\section{Experiment 2}

One potentially troublesome aspect of the action effect paradigm is that the action and no action condition differ in two potentially meaningful ways. One is the manipulation of interest in the current study; the go/no-go stimulus is responded to in the action condition and not responded to in the no-action condition. The other is that in the action condition the participants see a color word followed by a matching colored stimulus and then a search display also containing that color, but in the no action condition there is a mismatch in the stimulus colors across the three stimulus displays (but see: Buttaccio \& Hahn, 2011, Experiments 3 and 4). This means that the validity effect in the no action condition may be related to stimulus specific priming building up across the three stimulus displays rather than whether participants respond to the go/ no-go stimulus. An additional factor maybe a Stroop-like effect (Stroop, 1935) in which seeing a given color word may affect the processing of the color circle differently depending on whether the color word and color are congruent or incongruent. In the current experiment, we tested 
for these possibilities. We used a procedure where participants observed both the action and no action stimulus display sequences from the replication condition of Experiment 1 but never made a response in either case. In addition, we included catch trials where the go/no-go stimulus was red (with red never appearing in any other display) and the participants needed to make a spacebar response. This was done to ensure that participants continued attending to the go/no-go stimuli. If the action effect results from stimulus asymmetries between the action and no-action condition, then it should have been present in this experiment. If, however, an action is necessary for generating the action effect, then there should be no validity effects in this experiment. This also allows us to provide an additional test of the feature integration hypothesis of the action effect. Previous work has shown that a "no response" code can be integrated with stimuli (Kühn \& Brass, 2010; Kühn et al., 2009; Röttger \& Haider, 2016). If that is the case, then the feature integration hypothesis predicts that RTs will be slower in the condition in which a response is withheld from the go/no-go stimulus and that stimulus contains the target.

\section{Method}

Participants Fifteen undergraduates from the University of Toronto participated in the experiment and were compensated with course credit. All participants provided informed consent, were naïve to the experiment's purpose, and reported normal or corrected-to-normal visual acuity and color vision.

Stimuli and apparatus The stimuli and apparatus were the same as Experiment 1 with the exception that we added a red [CIE: luminance $=10.4 \mathrm{~cd} / \mathrm{m}^{2}, \mathrm{x}=0.581, \mathrm{y}=0.343$ ] go/no-go stimulus rather than only having blue or yellow circles.

Procedure The procedure matched that of the replication condition from Experiment 1 with the following exceptions. When the go/no stimulus was blue or yellow, the participants did not respond, regardless of whether it matched the color word or not. Instead, we included catch trials where the go/no-go stimulus was red and instructed the participants to press the spacebar when the go/no-go stimulus was red.

Design Participants first completed a set of ten practice trials, which were repeated if the experimenter deemed it necessary. This was followed by 240 randomly ordered test trials generated by repeating the color word ("YELLOW" or "BLUE"), go/no-go stimulus color (yellow, blue, or red), target placeholder color (yellow or blue), and target line tilt 10 times each. For analyses, we coded trials by whether the color word and go/no-go stimulus matched or mismatched and whether the go/no-go stimulus validly or invalidly cued the target placeholder color producing a 2 (match or mismatch) $\times 2$ (valid or invalid) design. Catch trials were not analyzed other than as a test of whether the participants were attending the go/no-go stimulus.

\section{Results}

Accuracy on the catch trials was high $(M=97.6 \%, S D=$ $3.9 \%$ ), indicating that participants did attend the go/no-go stimulus. We trimmed all trials where participants made an error or responded in less than $100 \mathrm{~ms}(5.5 \%)$. We submitted the data to a 2 (match or mismatch) $\times 2$ (valid or invalid) repeated measures ANOVA with RT as the dependent measure. The results are visualized in Fig. 3. The ANOVA indicated no main effect of match, $F(1,14)=1.451, M S E=$ 256.844, $p=.248, \eta_{p}^{2}=0.094$, no main effect of validity, $F(1,14)<1, M S E=337.441, p=0.404, \eta_{p}^{2}=0.050$, and no interaction between the two factors, $F(1,14)<1, M S E=$ 832.960, $p=0.548, \eta_{p}^{2}=0.026$, indicating the lack of any stimulus-based priming effect.

As with Experiment 1, we then conducted the Bayesian analysis to quantify the strength of evidence for the possible models. The analysis provided clear evidence in support of the null hypothesis over the action effect $(B F=20.83)$. Furthermore, the analysis provided some evidence in favor of null hypothesis compared with the match main effect $(B F=2.83)$, the validity main effect $(B F=2.96)$, and independent contributions from both main effects $(B F=8.48)$.

For the corresponding error data analysis, neither of the main effects nor the interaction neared significance, $F_{S}<1, p s>0.586$, which was confirmed by a Bayesian analysis which preferred the null hypothesis $(B F=$ 6.34), aligning with both the frequentist and Bayesian RT analysis results.

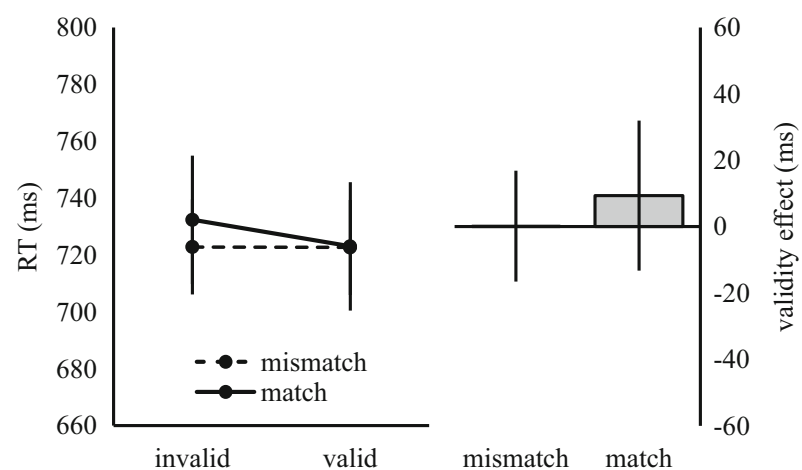

Fig. 3 Results from Experiment 2. Error bars represent the 95\% confidence interval of validity effect in both plots. 


\section{Discussion}

The results from Experiment 2 indicate that the action effect cannot be explained by color matching or mismatching differences between the action and no action conditions. The lack of effects in this experiment supports the notion that the action specifically generated the validity effect of the replication condition in Experiment 1. That is not to say that stimulus-based priming does not exist nor that only actions can lead to validity effects in paradigms, such as the current one, but it does indicate that stimulus-based priming does not account for the action effect. The lack of priming is consistent with previous studies that have failed to find stimulus-based priming when the stimulus preceding the search display does not require any response and does not involve any selective pressure (Goolsby \& Sazuki, 2001; Meeter \& Olivers, 2006; Yashar, Makovski, \& Lamy, 2013). There are some exceptions where an isolated, central cue has produced validity effects in a subsequent visual search (Ansorge \& Becker, 2012; Theeuwes, Reimann, \& Mortier, 2006). While those studies were similar to the current experiment in that they used a central cue that was not responded to before a visual search, there are many methods differences that may have led to the discrepant results. For example, they varied in the presence/absence of singletons, the types of responses the visual search required, and the relevance of the cue for predicting upcoming target attributes. These differences make it difficult to compare their results with results from action effect experiments. Experiment 2 also provides further evidence that feature integration processes do not underlie the action effect. Given that responses were withheld from the go/no-go stimulus, feature integration would predict that a no response code would be integrated with that stimulus. This should lead to slower responses to targets in that stimulus during the search phase. Because it does not, it appears more likely that attentional weighting underlies the action effect.

\section{General discussion}

When a visual search follows a go/no-go task, responses in the visual search task are faster when the target is contained within a go stimulus than if it appears elsewhere. In contrast, in the no-go condition, responses in the visual search task tend to be equally fast regardless of where the target appears. The goal of present study was to test the extent to which the biased competition principle can account for the action effect (Buttaccio \& Hahn, 2011; Weidler \& Abrams, 2014). We began by replicating this phenomenon using only two, rather than six, stimulus colors. We replicated the validity effect and, surprisingly, observed a robust inverse validity effect; when a response was withheld from a stimulus faster responses were observed in the visual search if that stimulus contained a distractor compared to when it contained a target. We compared the data from this replication to a similar condition in which we presented no distractor during the search. This manipulation removed both the validity and inverse validity effects. Instead, a Bayesian analysis strongly preferred the null hypothesis model over there being any effects present. These two findings suggest that the action effect is driven by a selection bias for the responded to stimulus, consistent with the biased competition hypothesis. Specifically, responding to a stimulus increased its attentional weight, causing a selection bias towards that stimulus. In Experiment 2, we demonstrated that stimulus-based priming is insufficient to generate the observed validity effects. Based on these experiments, we suggest that the action effect is primarily a result of the first response increasing the stimulus's attentional weight, causing it to be biased in the subsequent competition for attentional selection.

Both Buttaccio and Hahn (2011) and Weidler and Abrams (2014) suggested the possibility that the action effect results from increased weighting of the responded to stimulus's features. Buttaccio and Hahn (2011) argued that responding to a stimulus strengthens the memory trace of it, which increases the attentional weights assigned to those features. Conversely, Weidler and Abrams (2014) pointed out two other possible mechanisms that could lead to increased attentional weighting. One possibility is an event file (Hommel, 1998) stored in working memory increases the attentional weights of those features, which biases visual search (Olivers, Meijer, \& Theeuwes, 2006; Olivers, Peters, Houtkamp, \& Roelfsema, 2011). The other possibility is repetition priming of stimulus features (Maljkovic \& Nakayama, 1994; Wolfe, Butcher, Lee, \& Hyle, 2003; for a review: Kristjánsson \& Campana, 2010) referring to the general finding that visual search response times across trials decrease when targets are repeated because the target's feature weights are increased when they are responded to (Ásgeirsson et al., 2015). We next consider the implications of each of these hypotheses given the current data and previous research.

\section{Previous hypotheses}

The first consideration regards the implications of responses strengthening the target stimulus's memory strength. Specifically, previous research has demonstrated that responses to targets associated with stronger memory traces are speeded relative to weaker memory traces (Clifton \& Cruse, 1977; Dosher, 1984; Murdock, 1985). Given that evidence, responding to a previously responded to stimulus should be faster regardless of the presence or absence of distractors. Experiment 1's no search condition clearly demonstrates that this is not the case. Responding to the first stimulus did not modulate how quickly participants responded to the second stimulus. Main effects of action have been found in other action effect experiments, however. In our replication 
condition, we found faster responses following actions, as did Buttaccio and Hahn (Experiment 2; 2011). In contrast, Buttaccio and Hahn's Experiment 4 and Weidler and Abrams's (2014) Experiment 1 found slower responses in the action condition. Evidence that responding to stimuli increases its memory trace, which would lead to faster responses following actions, is not consistently found.

The second consideration is the possibility that the visual system integrates the first stimulus and response into an event file (Hommel, 1998). Holding this event file in working memory biases the visual search to the stimulus features included in the event file (Olivers et al., 2006, 2011). To date, no action effect experiments directly address this possibility. Note, however, that studies demonstrating interactions between working memory and attention typically require the participants' active maintenance of the item in working memory. In contrast, no memory maintenance is required in action effect studies. Furthermore, task-irrelevant "accessory items" do not typically interact with attentional selection (Olivers et al., 2011). The action effect does not require working memory maintenance, and the first stimulus's features are irrelevant to the second task. Therefore, a working memory and attention interaction is an unlikely mechanism to be underlying the action effect.

Relatedly, a recent hypothesis proposes that the action effect is due to a "respond" code being integrated with the go/ no-go stimulus (Wang et al., 2017). When the search target is in that stimulus, responding is then facilitated. If there is a mismatch, however, responding is hindered. This response feature integration explanation provides a good account for the validity effect in the action condition, but what about the inverse validity effect in the no-go condition? The respond code explanation remains viable if one assumes that a "no respond" code also can be integrated with the go/no-go stimulus, an assumption with some support in the literature (Kühn \& Brass, 2010; Kühn et al., 2009, Röttger \& Haider, 2016). Thus, there would be a cost for responding to the matching stimulus in the search task, because it is bound with a "no respond" code. This explanation also would predict validity effects in the no search condition, because the presence or absence of a distractor is irrelevant to response feature integration. Therefore, both the validity and inverse validity effects should have been found in the no search condition of Experiment 1. Furthermore, because the responses in Experiment 2 needed to be withheld from the blue/yellow stimuli, there should have been costs for responding to targets in that color placeholder (because of the "no respond" binding). That no validity effects were found in the no search condition of Experiment 1 or Experiment 2 indicates that the action effect is not due to feature integration benefits/costs between the go/no-go stimulus and the search targets. Rather, it appears to be dependent on the actions increasing the attentional weight of stimuli, consistent with the biased competition account.
While the memory trace and event file explanations have problems explaining the action effect, we are aware of no such difficulties for the repetition priming hypothesis, given the existing data. Rather, the main issues with this possibility are: what is the effect of withholding a response for repetition priming, and does repetition priming occur when the first stimulus is isolated? Most repetition priming experiments require responses on each trial and studies that have manipulated responding report inconsistent repetition priming effects (Goolsby \& Sazuki, 2001; Kristjánsson, Saevarsson, \& Driver, 2013; Yashar et al., 2013). One suggestion to reconcile the inconsistency has been that these studies have confounded responding with selection pressure (Ásgeirsson et al., 2015). According to this view, repetition priming will occur any time there is competition for attentional resources (Meeter \& Olivers, 2006), regardless of whether a response is made, because selecting the target increases the task relevance while distractor feature task relevance values are decreased. In a manner of speaking, the action effect is inconsistent with this claim; there is no selective pressure for the first target.

We suggest that associating a stimulus with a response similarly increases its attentional weight such that the visual system prioritizes it for selection. Indirect evidence for this comes from visual search experiments in which response compatible targets are prioritized in search. For example, when searching for a target with a specific orientation that must be grasped, fewer eye movements are made to targets with the wrong orientation compared with when targets must be pointed to (Bekkering \& Neggers, 2002). Likewise, size determined targets are found faster than luminance determined targets when grasping movements must be made, whereas the reverse is true when pointing movements must be made (Wykowska \& Schubö, 2012; Wykowska, Schubö, \& Hommel, 2009). Whereas those studies show that more inherently response compatible target dimensions are prioritized in search, the action effect seems to result from quickly formed $\mathrm{S}-\mathrm{R}$ associations causing one stimulus to become action compatible, receive increased attentional weight, and bias attentional selection.

\section{Other considerations}

In discussing repetition priming, Ásgeirsson et al. (2015) argue that distracting items have their feature values reduced. If withholding a response has the same effect, then that stimulus will lose a competition with a neutral stimulus, producing the inverse validity effect found here. If that is the case, why is the inverse validity effect not found in most action effect experiments? One possibility is the change from six to two stimulus colors in the current study. With only two stimulus possibilities, it is possible the visual system simultaneously decreases the ignored stimulus's feature values while increasing the alternative stimulus's feature values. This scenario is less 
feasible when there are six possible stimuli compared with the current study that used two colors. Experiment 4 from Weidler and Abrams is inconsistent with this claim, because they use six stimulus colors and find an inverse validity effect. In that experiment, they determined the go/no-go response by having participants withhold their response if an " $\mathrm{X}$ " appeared within the go/no-go stimulus. This creates a slightly different situation in the go/no-go phase in which the go response makes the, for example, blue circle task-relevant, but the no-go response is made to the " $\mathrm{X}$ " that is on a task-irrelevant circle. This may lead to something akin to a partial repetition cost (Hommel, 1998) when a response is required to another blue circle with a slightly different black line object on it. If that is the case, the inverse validity effect from Weidler and Abrams is driven by a different mechanism than the one found in Experiment 1 here and may explain why they found one using six stimulus colors.

Also noteworthy is that biased competition models of selection argue that attentional weights are used to prioritize stimuli for access into the capacity limited visual working memory so that they can undergo further processing (Bundesen, 1998). Given that visual working memory can hold around four items (Cowan, 2001; Luck \& Vogel, 1997), it is possible to argue that biased competition models would not predict the action effect when only two items are used as there is adequate visual working memory capacity to process both items. The mitigating circumstance in action effect experiments, we believe, is that the search items appear at peripheral locations (10-12 ${ }^{\circ}$ visual angle from center), so that it is necessary to search the items one-by-one to discriminate the line orientation. This proposal predicts that larger search displays should produce larger action effects and the available evidence supports this prediction. On average, six-item displays resulted in interactions with a mean $F=29.93$ and a mean action effect of $110 \mathrm{~ms}$, whereas experiments that have used two-item searches have resulted in interactions with a mean $F=16.22$ and a mean action effect of $58 \mathrm{~ms}$ (Buttaccio \& Hahn, 2011; Weidler \& Abrams, 2014). Another prediction of this hypothesis is that if the stimuli are presented such that both lines can be discriminated within one fixation, the action effect should be reduced or eliminated. No data that we know of exist yet to evaluate this claim.

Finally, it is worth noting the relationship between the action effect and the rapidly expanding field examining how reward biases attentional allocation. Within these studies, participants typically go through a learning phase in which specific stimuli are associated with high and low rewards. In a second phase, the degree to which these stimuli capture attention is measured. These tests show that highly rewarded stimuli capture attention more than lowly rewarded stimuli. These effects can be found with long training phases (Anderson, Laurent, \& Yantis, 2011) or trial-by-trial (Hickey, Chelazzi, $\&$ Theeuwes, 2014) using cash or nonmonetary rewards
(Rajsic, Perera, \& Pratt, 2016). Explicitly rewarding stimuli, as others have noted (Awh, Belopolsky, \& Theeuwes, 2012; Nakayama \& Martini, 2011), is only one of a variety of methods that can produce attentional biases. For example, attentional biases can be caused by target frequency (Hick, 1952; Hyman, 1953), associative learning of response effects (Gozli et al., 2014), and stimulus-pain learning (Koster, Crombez, Van Damme, Verschuere, \& De Houwer, 2005). The action effect demonstrates an additional method of reward: merely responding to a stimulus. This is an ecologically intuitive claim, as previously responded to items are more likely to be behaviorally relevant in the current situation. Of course, this is an empirical claim that requires further testing. Furthermore, we suspect that above-mentioned methods for generating attentional biases also could generate the bias observed in modified versions of the current paradigm. If true and a common mechanism underlies the different biases, it predicts interactions between the action effect and other manipulations that bias the degree to which a stimulus captures attention such as reward learning, high frequency targets, and relative target/distractor salience.

\section{References}

Abrams, R. A., \& Weidler, B. J. (2013). Trade-offs in visual processing for stimuli near the hands. Attention, Perception, \& Psychophysics, 76, 383-390.

Anderson, B. A., Laurent, P. A., \& Yantis, S. (2011). Value-driven attentional capture. Proceedings of the National Academy of Sciences, 108, 10367-10371.

Ansorge, U., \& Becker, S. I. (2012). Automatic priming of attentional control by relevant colors. Attention, Perception, \& Psychophysics, 74, 83-104.

Ásgeirsson, Á. G., Kristjánsson, Á., \& Bundesen, C. (2015). Repetition priming in selective attention: A TVA analysis. Acta Psychologica, 160, 35-42.

Awh, E., Belopolsky, A. V., \& Theeuwes, J. (2012). Top-down versus bottom-up attentional control: A failed theoretical dichotomy. Trends in Cognitive Sciences, 16, 437-443.

Bekkering, H., \& Neggers, S. F. W. (2002). Visual search is modulated by action intentions. Psychological Science, 13, 370-374.

Bichot, N. P., Rossi, A. F., \& Desimone, R. (2005). Parallel and serial neural mechanisms for visual search in macaque area V4. Science, 308, 529-534.

Blakemore, S. J., Wolpert, D. M., \& Frith, C. D. (1998). Central cancellation of self-produced tickle sensation. Nature Neuroscience, 1, 635-640.

Bundesen, C. (1998). A computational theory of visual attention. Philosophical Transactions of the Royal Society of London. Series B, Biological Sciences, 353, 1271-1281.

Buttaccio, D. R., \& Hahn, S. (2011). The influence of action on visual search: Behavioral response toward stimuli modifies the selection process. Attention, Perception, \& Psychophysics, 73, 1453-1466.

Cardoso-Leite, P., Mamassian, P., Schütz-Bosbach, S., \& Waszak, F. (2010). A new look at sensory attenuation. Action-effect 
anticipation affects sensitivity, not response bias. Psychological Science, 21, 1740-1745.

Clifton, C., Jr., \& Cruse, D. (1977). Time to recognise tones: Memory scanning or memory strength? The Quarterly Journal of Experimental Psychology, 29, 709-726.

Cowan, N. (2001). The magical number 4 in short term memory: A reconsideration of storage capacity. Behavioral and Brain Sciences, 24, 87-186.

Desimone, R., \& Duncan, J. (1995). Neural mechanisms of selective visual attention. Annual Review of Neuroscience, 18, 193-222.

Dosher, B. A. (1984). Degree of learning and retrieval speed: Study time and multiple exposures. Journal of Experimental Psychology. Learning, Memory, and Cognition, 10, 541-574.

Firestone, C., \& Scholl, B. J. (2015). Cognition does not affect perception: Evaluating the evidence for "top-down" effects. Behavioral and Brain Sciences, 4629, 1-77.

Fodor, J. A. (1983). The modularity of mind: An essay on faculty psychology. MIT press.

Goolsby, B. A., \& Sazuki, S. (2001). Understanding priming colorsingleton search: Roles of attention at encoding and "retrieval". Perception \& Psychophysics, 63, 929-944.

Gozli, D. G., Aslam, H., \& Pratt, J. (2016). Visuospatial cueing by selfcaused features: Orienting of attention and action-outcome associative learning. Psychonomic Bulletin \& Review, 23, 459-467.

Gozli, D. G., Moskowitz, J. B., \& Pratt, J. (2014). Visual attention to features by associative learning. Cognition, 133, 488-501.

Gozli, D. G., West, G. L., \& Pratt, J. (2012). Hand position alters vision by biasing processing through different visual pathways. Cognition, $124,244-250$.

Haig, B. D. (2005). An abductive theory of scientific method. Psychological Methods, 10, 371-388.

Hick, W. E. (1952). On the rate of gain of information. Quarterly Journal of Experimental Psychology, 4, 11-26.

Hickey, C., Chelazzi, L., \& Theeuwes, J. (2014). Reward-priming of location in visual search. PLOS ONE, 9.

Hilchey, M. D., Rajsic, J., Huffman, G., \& Pratt, J. (2017). Intervening response events between identification targets do not always turn repetition benefits into repetition costs. Attention, Perception, \& Psychophysics, 1-13.

Hommel, B. (1998). Event files: Evidence for automatic integration of stimulus-response episodes. Visual Cognition, 5, 183-216.

Hommel, B. (2005). How much attention does an event file need? Journal of Experimental Psychology: Human Perception and Performance, 31, 1067-1082.

Hommel, B., Müsseler, J., Aschersleben, G., \& Prinz, W. (2001). The theory of event coding (TEC): A framework for perception and action planning. The Behavioral and Brain Sciences, 24, 849-878.

Huffman, G., Al-Aidroos, N., \& Pratt, J. (2017). Salience drives nonspatial feature repetition effects in cueing tasks. Attention, Perception, \& Psychophysics, 79, 212-222.

Huffman, G., Gozli, D. G., Welsh, T. N., \& Pratt, J. (2015). Hand position influences perceptual grouping. Experimental Brain Research, 233, 2627-2634.

Hyman, R. (1953). Stimulus information as a determinant of reaction time. Journal of Experimental Psychology, 45, 188-196.

JASP Team. (2017). JASP (Version 0.8.1.1)[Computer software].

Kleiner, M., Brainard, D., Pelli, D., Ingling, A., Murray, R., \& Broussard, C. (2007). What's new in Psychtoolbox-3. Perception, 36, 1.

Koster, E., Crombez, G., Van Damme, S., Verschuere, B., \& De Houwer, J. (2005). Signals for threat modulate attentional capture and holding: Fear-conditioning and extinction during the exogenous cueing task. Cognition \& Emotion, 19, 771-780.

Kristjánsson, Á., \& Campana, G. (2010). Where perception meets memory: A review of repetition priming in visual search tasks. Attention, Perception, \& Psychophysics, 72, 5-18.
Kristjánsson, Á., Saevarsson, S., \& Driver, J. (2013). The boundary conditions of priming of visual search: From passive viewing through task-relevant working memory load. Psychonomic Bulletin \& Review, 20, 514-521.

Kühn, S., \& Brass, M. (2010). The cognitive representation of intending not to act: Evidence for specific non-action-effect binding. Cognition, 117, 9-16.

Kühn, S., Elsner, B., Prinz, W., \& Brass, M. (2009). Busy doing nothing: Evidence for nonaction-effect binding. Psychonomic Bulletin \& Review, 16, 542-549.

Luck, S. J., \& Vogel, E. K. (1997). The capacity of visual working memory for features and conjunctions. Nature, 390, 279-281.

Maljkovic, V., \& Nakayama, K. (1994). Priming of pop-out: I. Role of features. Memory \& Cognition, 22, 657-672.

Meeter, M., \& Olivers, C. N. L. (2006). Intertrial priming stemming from ambiguity: A new account of priming in visual search. Visual Cognition, 13, 202-222.

Murdock, B. B. (1985). An analysis of the strength-latency relationship. Memory \& Cognition, 13, 511-521.

Müsseler, J., \& Hommel, B. (1997). Blindness to response-compatible stimuli. Journal of Experimental Psychology: Human Perception and Performance, 23, 861-872.

Nakayama, K., \& Martini, P. (2011). Situating visual search. Vision Research, 51, 1526-1537.

Olivers, C. N. L., Meijer, F., \& Theeuwes, J. (2006). Feature-based memory-driven attentional capture: Visual working memory content affects visual attention. Journal of Experimental Psychology: Human Perception and Performance, 32, 1243-1265.

Olivers, C. N. L., Peters, J., Houtkamp, R., \& Roelfsema, P. R. (2011). Different states in visual working memory: When it guides attention and when it does not. Trends in Cognitive Sciences, 15, 327-334.

Proctor, R. W., \& Cho, Y. S. (2006). Polarity correspondence: A general principle for performance of speeded binary classification tasks. Psychological Bulletin, 132, 416-442.

Raftery, A. E. (1995). Bayesian model selection in social research. Sociological Methodology, 25, 111-163.

Rajsic, J., Perera, H., \& Pratt, J. (2016). Learned value and object perception: Accelerated perception or biased decisions?. Attention, Perception, \& Psychophysics, 1-11.

Röttger, E., \& Haider, H. (2016). Investigating the characteristics of "not responding": Backward crosstalk in the PRP paradigm with forced vs. free no-go decisions. Psychological Research, 1-15.

Stroop, J. R. (1935). Studies of interference in serial verbal reactions. Journal of Experimental Psychology, 18, 643-662.

Taylor, J. E. T., Gozli, D. G., Chan, D., Huffman, G., \& Pratt, J. (2015). A touchy subject advancing the modulated visual pathways account of altered vision near the hand. Translational Neuroscience, 6, 1-7.

Taylor, J. E. T., \& Witt, J. K. (2014). Altered attention for stimuli on the hands. Cognition, 133, 211-225.

Taylor, J. E. T., Witt, J. K., \& Sugovic, M. (2011). When walls are no longer barriers: Perception of wall height in parkour. Perception, 40, 757-760.

Theeuwes, J., Reimann, B., \& Mortier, K. (2006). Visual search for featural singletons: No top-down modulation, only bottom-up priming. Visual Cognition, 14, 466-489.

Wang, F., Sun, J., Sun, P., Weidler, B. J., \& Abrams, R. A. (2017). Influence of simple action on subsequent manual and ocular responses. Attention, Perception, \& Psychophysics, 79, 389-395.

Weidler, B. J., \& Abrams, R. A. (2014). Decomposing the action effect: How simple actions affect subsequent perception. Attention, Perception, \& Psychophysics, 76, 1242-1252.

Weidler, B. J., \& Abrams, R. A. (2017). Simple actions influence pop-out search. Visual Cognition, 1-14.

Witt, J. K., Linkenauger, S. A., \& Proffitt, D. R. (2012). Get me out of this slump! Visual illusions improve sports performance. Psychological Science, 23, 397-399. 
Wolfe, J. M., Butcher, S. J., Lee, C., \& Hyle, M. (2003). Changing your mind: On the contributions of top-down and bottom-up guidance in visual search for feature singletons. Journal of Experimental Psychology: Human Perception and Performance, 29, 483-502.

Wykowska, A., \& Schubö, A. (2012). Action intentions modulate allocation of visual attention: Electrophysiological evidence. Frontiers in Psychology, 3, 1-15.
Wykowska, A., Schubö, A., \& Hommel, B. (2009). How you move is what you see: Action planning biases selection in visual search. Journal of Experimental Psychology: Human Perception and Performance, 35, 1755-1769.

Yashar, A., Makovski, T., \& Lamy, D. (2013). The role of motor response in implicit encoding: Evidence from intertrial priming in pop-out search. Vision Research, 93, 80-87. 